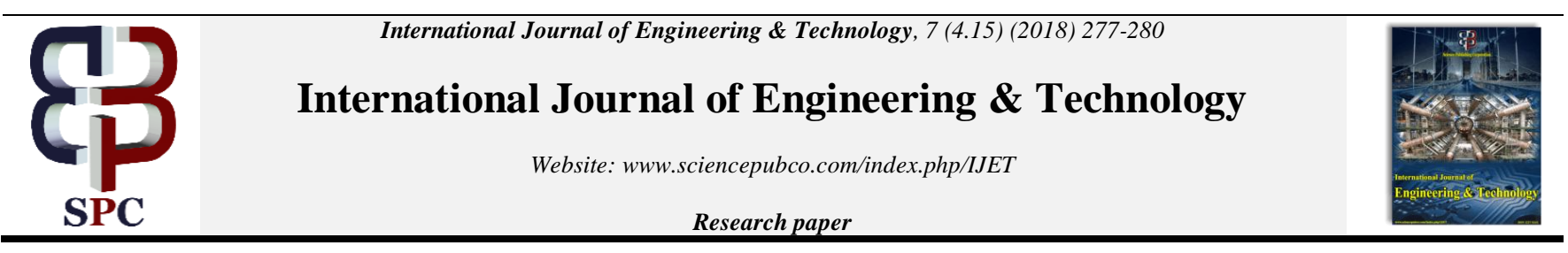

\title{
Crop Recommender System for the Farmers using Mamdani Fuzzy Inference Model
}

\author{
Madhusree Kuanr *, Bikram Kesari Rath, Sachi Nandan Mohanty \\ Gandhi Institute for Technology, Bhubaneswar, India \\ *Corresponding Author E-mail: madhu.kuanr@gmail.com
}

\begin{abstract}
Recommender systems provide suggestions to the users for choosing particular items from a large pool of items. The purpose of this study is to design a collaborative recommender system for the farmers for recommending giving prior idea regarding a crop which is suitable according to the location of the farmer based on weather condition of the previous months. The proposed system also recommends other seeds, pesticides and instruments according to the preferences in farming and location of the farmers while purchasing the seeds through online. It uses cosine similarity measure to find the similar user according the location of the farmer and fuzzy logic for predicting the yield of rice crop for Kharif season in state Odisha, India. The proposed system is implemented in Mamdani Fuzzy Inference model. The results reveal that it provides prior idea regarding a crop before sowing of seeds.
\end{abstract}

Keywords: Collaborative Recommender system, cosine similarity, fuzzy logic, Mamdani Fuzzy Inference model

\section{Introduction}

Recommender systems are the personalization tools which use the opinions of a community of users to help individual by recommending a set of items from a potentially overwhelming set of choices [1]. It has changed the way inanimate websites communicate with their users. The different traditional recommender systems are collaborative, content-based, knowledge-based and hybrid recommender systems. Collaborative filtering methods are based on collecting and analyzing a large amount of information on users' behaviors, activities or preferences and predicting user's expectations based on their similarity to other users. Collaborative filtering methods are further categorized as memory-based and model based collaborative filtering. An eminent example of memory-based approaches is user-based algorithm [2] and that of model-based approaches is Kernel-Mapping Recommender [3]. Content-based recommender systems recommend items to the users based on correlation between the content of items and the user preferences [4]. In these systems, the user is recommended items similar to the items the user preferred in the past [5]. Text documents are widely used as source of information source. To make recommendations, content- based systems mostly work by calculating how strongly a not yet seen item is similar to items the active user has liked in the past. Regarding the users specific tasks knowledge based recommendation can address problem by using a model of knowledge. A knowledge-based recommender system is based on inferences about a user's requirements and choices. This knowledge will sometimes contain explicit functional knowledge about how certain product features meet user need [6][7]. A hybrid approach, combines collaborative filtering and contentbased filtering. Hybrid approaches can be applied in several ways: by making content-based and collaborative-based predictions independently and then binding them; by adding content-based capabilities to a collaborative-based approach (and vice versa); or by merging the approaches into one model for a complete review of recommender systems [8]. Several empirical studies suggest that the performance of the hybrid with the pure collaborative methods, content-based methods and demonstrate that the hybrid methods can provide more accurate recommendations than pure approaches. These methods can also be used to overcome cold start and the sparsity problem. The goal of this paper is to provide affordable, personal and accurate recommendations.

\section{Literature Survey}

Recommender System helps the user for selecting items based on their personal preferences. A survey on the recommender system describes the different methods of recommender system like collaborative, content-based and hybrid recommender system along with their limitations and possible extensions which can improve the recommendation capabilities of these systems [9].The recommender system is proposed to recommend the right crops at right place to improve the yield using data mining [10]. A collaborative filtering recommender system is proposed on the basis of user's preferences, soil and weather condition which recommends suitable crop by using Statistic data analysis and predictive modeling [11].Machine learning also plays an important role to improve the capability of the recommender system. A recommender system for precision agriculture is proposed to recommend a crop by taking the report of soil testing as input with majority voting technique using support vector machines and artificial neural network [12]. A demand based recommender system is proposed which predicts the crop yield and price for a crop by applying sliding window non-linear regression technique on past data [13]. An agricultural information service recommender system is proposed which recommends page content of different websites to provide agriculture related information considering the preferences of the farmer [14]. Irrigation plays an 
important role in the productivity of a particular crop. A recommender system based on irrigation is proposed which recommends daily water requirement for a particular crop with a chosen location using a user friendly interface of traffic light system [15]. A recommender system on

precision agriculture using data mining techniques is proposed which recommends the farmers the right crop based on research data of their soil types, soil characteristics and crop yield [16]. A recommender system for farmers is proposed using production rate of past years and ecological and climatic information related to agriculture which helps the farmer for choosing the right crop [17]

\section{Research Gap}

Most of the time the farmers go for a particular crop being unaware of the climatic conditions of that time whether it will suit to the crop or not. It is some time impossible for the farmers to know the conditions of the soil through soil testing whether it will suit to the crop or not. Due to this reason some times they have to bear losses in the farming which in turn greatly affects our society. This paper proposes a recommender system which recommends whether the farmer should go for a particular crop or not considering the temperature, humidity and rainfall of the previous three months while visiting online shopping websites related to farming. The farmer has to give his location and one of the crop as preferences. The proposed system predicts the rate of yield of that particular crop based on the past information and then recommends whether the farmer should go for that crop or not. It also proposes fertilizers, pesticides and instruments for positive recommendation.

\section{Fuzzy Rule -based Systems}

Rule based systems play important roles in manipulating and storing knowledge to interpret information in useful ways. An inference engine (IE) and a knowledge base (KB) are considered as the two major components of a fuzzy rule-based system (FRBS). Knowledge can be represented in different ways. Natural language representation is the best way to represent the human knowledge. Fuzzy linguistic variables also plays vital role in representing the human knowledge. $\mathrm{KB}$ of a particular problem can be represented using these fuzzy linguistic variables. FRBS consists of IF-THEN rules using fuzzy linguistic variable and IE. $\mathrm{IE}$ is responsible for fuzzy inference processing to produce the output from the KRBS. Our proposed method uses twenty seven IF-THEN rules to infer the result for a given input. The schematic diagram of a fuzzy inference system is shown in the Fig.1.

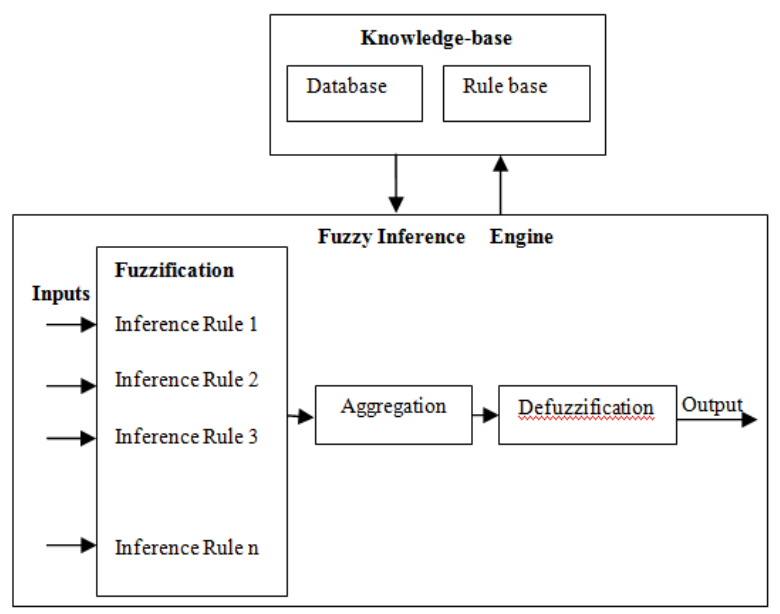

Fig.1: A Schematic diagram of a Fuzzy rule base system

\subsection{Linguistic Variables and Membership functions of Fuzzy Logic}

Information can be represented by linguistic variables in a form and precision appropriate for the problem. Linguistic variables are essential to fuzzy logic implementation. Linguistic variable has shown its challenging affect in complex non-linear applications .Linguistic variables reduces the overall computation of the application [18]. A fuzzy set A can be represented by a pair of values. The first value is the element of the fuzzy set and the second value is the membership value of the element in the fuzzy set. The membership value defines the degree to which the element is associated to the fuzzy set.

$A=\left\{\left(x_{1}, \mu\left(x_{1}\right)\right),\left(x_{2}, \mu\left(x_{2}\right)\right),\left(x_{3}, \mu\left(x_{3}\right)\right), \ldots,\left(x_{n}, \mu\left(x_{n}\right)\right)\right\}$

Where $x_{1}, x_{2}, x_{3}, \ldots, x_{n}$ are the elements of the fuzzy set in the universe of discourse $X$ and $\mu\left(x_{1}\right), \mu\left(x_{2}\right), \mu\left(x_{3}\right), \ldots, \mu\left(x_{n}\right)$ are the membership values of the these elements respectively. The range of the membership value lies between the range $[0,1]$. Different membership functions are available which can be used to produce the output for the system for a given input. The proposed methodology uses the triangular membership function to make the system simple. The degree of membership of triangular membership function can be defined as follows.

\subsection{Description of Input Variables}

The proposed methodology uses three fuzzy input variables, $\mathrm{V} 1=\{$ Temperature $\}, \mathrm{V} 2=\{$ Humidity $\} \quad$ and $\mathrm{V} 3=\{$ Rainfall $\}$.Each of the variable is represented using three linguistic terms such as $\operatorname{Low}(\mathrm{L}), \operatorname{Medium}(\mathrm{M})$ and $\operatorname{High}(\mathrm{H})$ as shown in the Fig. 2 .
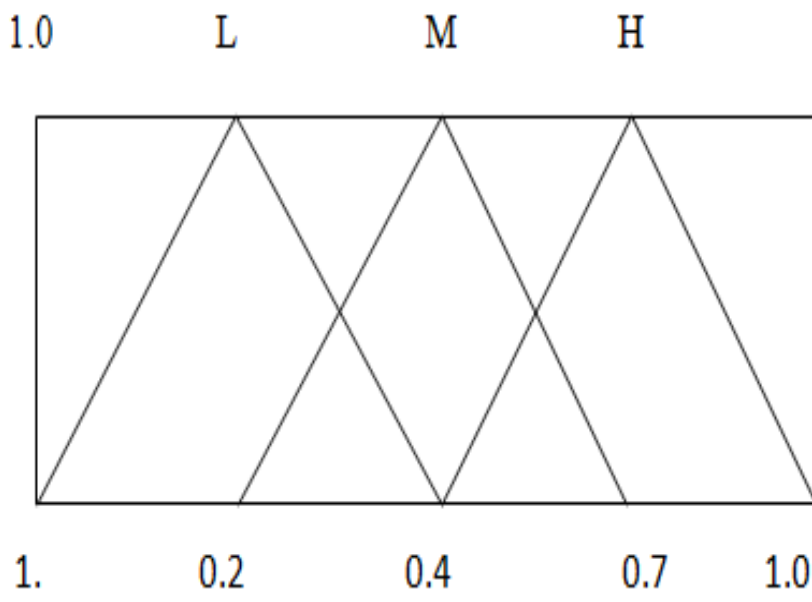

Fig.2: Fuzzy Input variables 'temperature', 'humidity', 'rainfall'

Table 1: Linguistic terms with their ranges

\begin{tabular}{|l|l|l|}
\hline Linguistic Terms & Ranges & Membership function \\
\hline Low(L) & {$[0.0,0.4]$} & Trimf \\
\hline Medium(M) & {$[0.2,0.7]$} & Trimf \\
\hline High(H) & {$[0.4,1.0]$} & Trimf \\
\hline
\end{tabular}

\subsection{Description of Output Variables}

The fuzzy output variable used for the proposed system is rice yield and the linguistic terms associated with this variable are Low and High as shown in Fig.3.
1.0
Low

High 


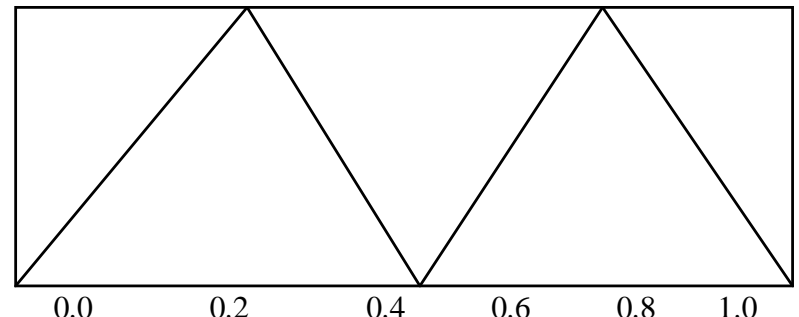

Fig.3: Fuzzy output variables with membership function distribution

The rules plays major role towards the strength of the FRBS. The rules state the relationship between the input and output variables and represents the knowledge base of the FRBS of a system. In the proposed method, three input variables with three linguistic terms are used and thus we have considered all the twenty seven rules in the FRBS. For instance, two rules are as follows:

IF $V_{1}$ is $L$ AND $V_{2}$ is $L$ AND $V_{3}$ is $L$ THEN output is Low.

Similarly,

IF $\mathrm{V}_{1}$ is $M$ AND $\mathrm{V}_{2}$ is $M$ AND $\mathrm{V}_{3}$ is $M$ THEN output is High

Table 2: Description of Linguistic terms of fuzzy variables

\begin{tabular}{|l|c|c|}
\hline Expression & Abbreviation & Index Representation \\
\hline Low & L & 0.25 \\
\hline Medium & M & 0.35 \\
\hline High & H & 0.55 \\
\hline
\end{tabular}

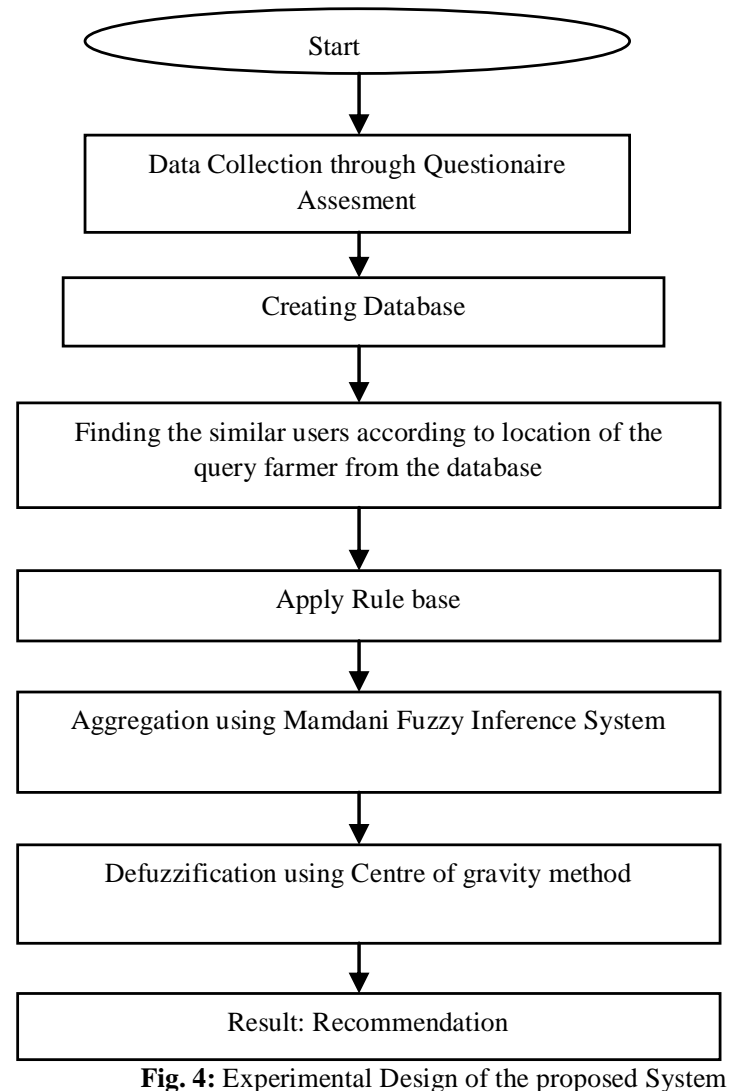

\section{Methodology}

\subsection{Dataset Description}

Four hundred $($ Male $=335$, Female=65) farmers from the district Jajpur of state Odisha, India have participated in this study. The questionnaire administrate to all the farmers. Each questionnaire contains twenty questions having rainfall, humidity, temperature, and yield of rice crops. The questionnaire has been asked in local language for better understanding of the respondents. All the respondents are having maximum qualification upper primary and belong to nuclear family. The data has been collected for Kharif season (June to December) from 2012 to 2017.

\subsection{Experimental Design}

The system takes the farmer's location(state \&district) and preferences like name of the crop and the season (Kharif/Rabi) as input and suggests whether the farmer should go for that crop in that season by predicting the yield of that product in that season. It will recommend the crop if the prediction of the yield of that crop found to be High. The cosine similarity measure is used to find the similar farmers in terms of location from the database. The system computes the cosine similarity of the querying farmer with all the farmers present in the database by using the following formula.

$$
\cos (d 1, d 2)=\frac{(d 1 . d 2)}{\|d 1\||| d 2 \|}
$$

Where (. ) indicates vector dot product and $\|d 1\|$ and $\|\mathrm{d} 2\|$ are the length of vectors $\mathrm{d} 1$ and $\mathrm{d} 2$ respectively.

The resultant farmers who are similar to the querying farmer form the database form the knowledge base with the rule base. The rule base consists of the twenty seven rules. Both of this database and rule base form the knowledgebase of FRBS. For the given inputs, the IE inferences by using the twenty seven inferences rules of FRBS. The Mamdani min-operator is used for aggregation process. The centre of gravity method is used for defuzzification process.

The processing of the fuzzy input variables to produce the output is shown in the Fig.5 .The system also proposes pesticides, fertilizers, instruments and other seeds for positive recommendations

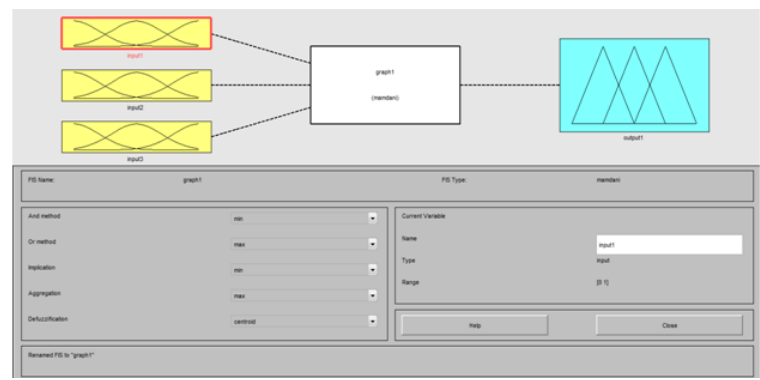

Fig. 5: Three input and Two output Fuzzy Mamdani Approach

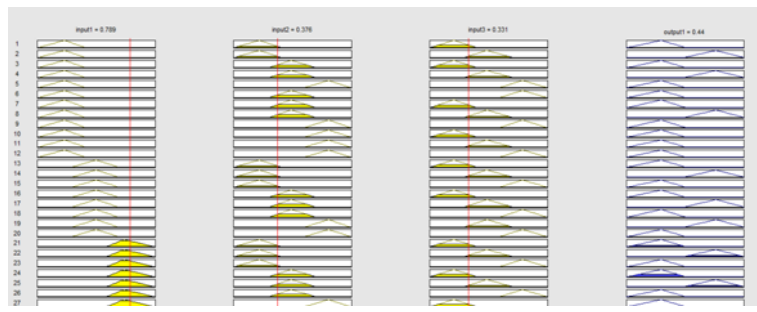

Fig. 6: Encoding rules of Fuzzy rule based system

\section{Results \& Discussion}

In this study we have designed 3 input 2 output Mamdani Fuzzy inference model for quantifying the yield of the rice corp. The three input variables are such as temperature, humidity and rainfall. Each input variables are having three linguistic terms (low, medium \& high). A set of twenty seven rules are generated as shown in the Fig.6. Our results revealed that, if the temperature is high and rain fall is high then the rice yield for the Kharif season in the location is low(See fig. 7(a)). Similarly, when the temperature is high and humidity is high then the yield is low (See 
fig. 7(b)). Whereas the humidity is medium and the rain fall is medium, then the yield of rice is high(See 7(c)).

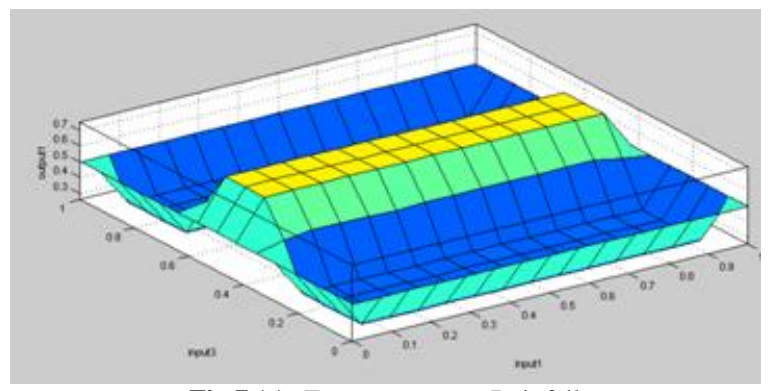

Fig.7 (a): Temperature vs. Rainfall

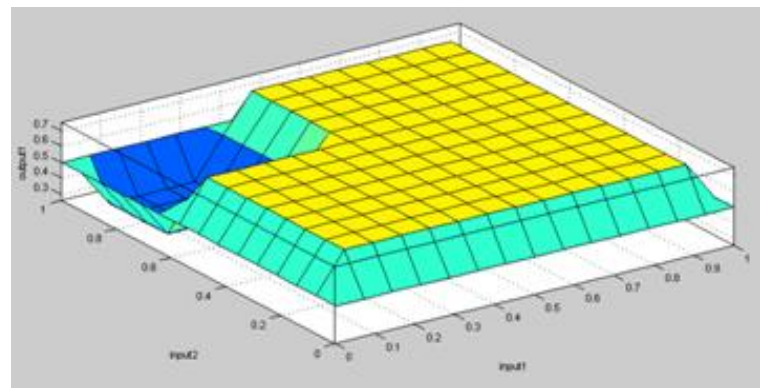

Fig.7 (b): Temperature vs. Humidity

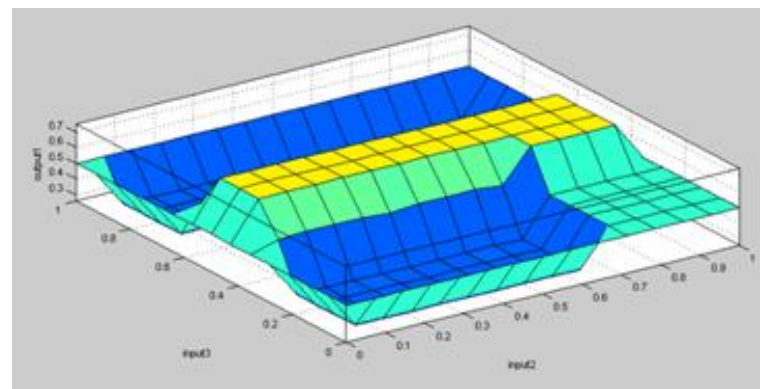

Fig.7 (c): Humidity vs. Rainfall

\section{Conclusion \& Future Work}

In Agriculture domain recommender systems play important role for choosing the best items to the users according to their preferences. The efficient functioning of the recommender systems help the farmers to fiddle with the changing climatic conditions. The proposed system gives the farmer a prior idea regarding the yield of a particular crop by predicting the yield according to the location of the farmer by using the past data of weather conditions and crop yield. This system can help the farmer from bearing loss in the farming by forecasting the crop yield prior to the sowing of seeds in the field. Hence the farmer can get idea regarding the yield of the crop prior to the ploughing and sowing of the seeds. Our studies focused only rice production and has not considered the other climatic condition like wind speed, acid rain etc. In future we will try to propose a recommender system which will consider most of the parameters which can affect the yield of the rice crop with the cost prediction.

\section{References}

[1] Ricci,F., Rokach,L. \& Shapira B.(2011). Introduction to Recommender Systems Handbook, Recommender Systems Handbook, Springer, 2011, pp. 1-35.

[2] Shardanand,U.\& Maes,P.(1995). Social Information Filtering: Algorithms for Automating 'Word of Mouth',Proc.conference on human factors in computing systems(CHI,95),pp.210-217
[3] Balabanovic,M. \& Shoham,Y.(1997). Fab: Content-Based, Collaborative Recommendation, Communications of the ACM, vol. 40, no. 3, pp. 66-72.

[4] "Facebook, Pandora Lead Rise of Recommendation Engines TIME". TIME.com. 27 May 2010. Retrieved 1 June 2015.

[5] Pu, P., Chen, L. \& Hu R. (2011).A user-centric evaluation framework for recmmender systems.In proceedings of the fifth ACM conference on recommender systems (pp.157-64). ACM

[6] Breese, J.S.; Heckerman, D. \& Kadie, C. (1998). Empirical Analysis of Predictive Algorithms for Collaborative Filtering (PDF) (Report). Microsoft Research.

[7] Ghazanfar, M. A., Prügel-Bennett, A., \& Szedmak, S. (2012). Kernel-mapping recommender system algorithms. Information Sciences, 208, 81-104.

[8] Burke, R. (2007). Hybrid web recommender systems. In The adaptive web (pp. 377-408). Springer, Berlin, Heidelberg.

[9] Adomavicius, G., \& Tuzhilin, A. (2005). Toward the next generation of recommender systems: A survey of the state-of-theart and possible extensions. IEEE Transactions on Knowledge \& Data Engineering, (6), 734-749.

[10] Univerity, S. P. P. SYSTEM FOR AGRICULTURE RECOMMENDATION USING DATA MINING.

[11] Reddy, K. A., \& Kumar, R. K. (2018). Recommendation System: A Collaborative Model for Agriculture

[12] Rajak, R. K., Pawar, A., Pendke, M., Shinde, P., Rathod, S., \& Devare, A. (2017). Crop recommendation system to maximize crop yield using machine learning technique. Int Res J Eng Technol, 4(12), 950-953.

[13] Raja, S. K. S., Rishi,R., Sundaresan,E. \& Srijit,V.(2017). Demand based crop recommender system for farmers. IEEE Technological Innovations in ICT for Agriculture and Rural Development (TIAR), Chennai, 2017, pp. 194-199.

[14] Yu, F., Zhang, Q., Luan, R., Zhang, J., \& Liu, X. (2013, July). Application and improvement of intelligent recommendation for Agricultural Information. In Natural Computation (ICNC), 2013 Ninth International Conference on (pp. 1077-1081). IEEE.

[15] Li, C., Dutta, R., Kloppers, C., D'Este, C., Morshed, A., Almeida, A., ... \& Aryal, J. (2013, November). Mobile application based sustainable irrigation water usage decision support system: An intelligent sensor CLOUD approach. In SENSORS, 2013 IEEE (pp. 1-4). IEEE.

[16] Pudumalar, S., Ramanujam, E., Rajashree, R. H., Kavya, C., Kiruthika, T., \& Nisha, J. (2017, January). Crop recommendation system for precision agriculture. In Advanced Computing (ICoAC), 2016 Eighth International Conference on (pp. 32-36). IEEE.

[17] Mokarrama.,M.J. \& Arefin.,M.S.(2017). RSF: A recommendation system for farmers. IEEE Region 10 Humanitarian Technology Conference (R10-HTC), Dhaka, 2017, pp. 843-850.

[18] Banks, W. (2008). Linguistic variables: Clear thinking with fuzzy logic. Byter craft limited. 